\title{
Silencing of glutaminase 1 resensitizes Taxol-resistant breast cancer cells to Taxol
}

\author{
AIQIN FU, ZE YU, YAOBO SONG and ENNING ZHANG \\ Department of Medical Oncology, Yantai Yantaishan Hospital, Yantai, Shandong 264000, P.R. China
}

Received May 7, 2014; Accepted January 2, 2015

DOI: $10.3892 / \mathrm{mmr} .2015 .3261$

\begin{abstract}
Taxol is a front-line chemotherapeutic agent for the treatment of patients with multiple types of tumor. However, resistance to Taxol remains one of the principal causes of cancer-associated mortality. Glutamine, which is metabolized via a glutaminase (GLS)-dependent process, termed glutaminolysis, is important in cell growth and metabolism. The present study reported a novel mechanism underlying Taxol resistance in breast cancer cells. By investigating the glutamine metabolism of breast cancer cells in response to treatment with Taxol in vitro, it was observed that Taxol induced the uptake of glutamine and the expression of GLS1. Notably, Taxol-resistant cancer cells exhibited upregulation in the metabolism of glutamine and expression of GLS1. In addition, overexpression of GLS1 rendered cancer cells resistant to Taxol, indicating that GLS1 may be the therapeutic target for overcoming Taxol resistance in clinical therapeutics. The results also demonstrated that knock-down of GLS1 using small interfering RNA, resensitized the Taxol-resistant breast cancer cells to Taxol.
\end{abstract}

\section{Introduction}

Taxol (paclitaxel) has emerged as an essential chemotherapeutic agent for the treatment of multiple types of tumor, including ovarian, prostate and non-small cell lung cancer (1-3). Taxol stabilizes the structure of microtubules by disrupting the dynamic equilibrium between soluble tubulin dimers and their polymerized form (3), therefore, cells treated with paclitaxel have problems with spindle assembly, cell division and chromosome segregation (3). Taxol is a potent anticancer drug, causing cell cycle arrest in the late G2 or mitotic phases (4). However, a significant percentage of patients develop drug resistance during the course of

Correspondence to: Dr Enning Zhang, Department of Medical Oncology, Yantai Yantaishan Hospital, 91 Jiefang Road, Yantai, Shandong 264000, P.R. China

E-mail: enningzhang@126.com

Key words: Taxol resistance, glutamine metabolism, glutaminase 1, chemoresistance, breast cancer treatment with Taxol, and the emergence of drug-resistant cancer cells has limited its clinical efficacy $(3,5)$. Therefore, it is imperative to develop novel strategies to reduce or overcome chemoresistance in cancer. The mechanism underlying Taxol resistance has been widely investigated. The overexpression of anti-apoptotic proteins, including survivin (6), myeloid cell leukemia 1 (7) and B cell lymphoma-2 (8), has been identified as an underlying mechanism contributing to the acquisition of Taxol resistance. In addition, evidence suggests that multiple upregulated oncogenes, including epidermal growth factor receptor 2 (ERBB2) $(9,10)$, Akt (11) and sarcoma (12), may be directly associated with drug resistance in cancer.

Cancer cells exhibit an increased dependency on aerobic glycolysis, fatty acid synthesis and glutaminolysis for proliferation (13). Glutaminolysis is the conversion of glutamine to glutamate, which is the first, and a rate-limiting step of glutamine utilization catalyzed by glutaminase (GLS). There are two isoforms of GLS: GLS1, which has been reported to be expressed in non-hepatic types of human tissue and tumor (14), and GLS2, a liver isoform, which is involved in the urea cycle (15). Glutaminolysis is not only an important procedure for providing adenosine triphosphate and the reducing equivalent, but is also utilized to meet biosynthetic, energetic and reductive needs for highly proliferating cells (16). It has been reported that cancer cells are particularly sensitive to glutamine deprivation and are unable to proliferate in culture in its absence (17).

The present study investigated the glutamine metabolism of breast cancer cells following treatment with Taxol in vitro. The glutamine uptake and the expression of GLS1 in breast cancer cells was measured in response to various concentrations of Taxol treatment. A Taxol-resistant cancer cell line was established and used to investigate the roles of GLS1 in Taxol-resistance through the regulation of glutamine metabolism. In addition, the present study aimed to determine whether GLS1 may be the therapeutic target for overcoming Taxol resistance, and to provide a novel insight into the cellular and molecular mechanisms involved in Taxol-resistant breast cancer.

\section{Materials and methods}

Cells and cell culture. The MDA-MB-231 and BT474 human breast cancer cell lines were purchased from American Type Culture Collection (Manassas, VA, USA). 
All cells were cultured in Dulbecco's modified Eagle's medium (DMEM)/F-12 (Mediatech, Inc., Manassas, VA, USA), supplemented with $10 \%$ fetal bovine serum (FBS; Life Technologies, Carlsbad, CA, USA) and 10\% penicillin/streptomycin (Life Technologies). The cells were cultured at $37^{\circ} \mathrm{C}$ in a humidified incubator with $95 \%$ air and $5 \% \mathrm{CO}_{2}$.

Antibodies and reagents. Mouse anti-GLS monoclonal antibody was purchased from Abcam (1:100; ab60709; Cambridge, MA, USA) and rabbit anti- $\beta$-actin polyclonal antibody was purchased from Cell Signaling Technology, Inc. (1:2,000; cat. no. \#4967; Danvers, MA, USA). A vector containing the wild-type open reading frame clone of the Homo sapiens protein, GLS1, was purchased from OriGene Technologies (cat. no. RC206265; Rockville, MD, USA). The siGLS1 and control siRNA were purchased from Ambion Life Technologies (Austin, TX, USA). Taxol was purchased from Sigma-Aldrich (St. Louis, MO, USA).

Generation of a Taxol-resistant cell line. The MDA-MB-231 cells (1x10\%/10 cm dish) were treated with gradually increasing concentrations of Taxol (50, 100, 150 and $200 \mathrm{nM})$ in regular cell culture conditions (DMEM/F-12 supplemented with $10 \% \mathrm{FBS}$ and $10 \%$ penicillin/streptomycin) for the selection of resistant cells. The medium containing taxol was refreshed every 4 days for 2 months, and then several resistant cell clones were developed from the parental cell line. The Taxol-resistant cell clones were pooled and used for subsequent experiments. Following selection and during the culture/passage of cells, the cells were treated with $200 \mathrm{nM}$ Taxol each month in order to maintain Taxol resistance.

Plasmid DNA and siRNA transfections. The transfections were performed using Oligofectamine ${ }^{\mathrm{TM}}$ Transfection reagent (Invitrogen Life Technologies, Carlsbad, CA, USA), according to the manufacturer's instructions. Briefly, between 0.5 and $1 \times 10^{6}$ cells were plated into 6 -well plates overnight at $37^{\circ} \mathrm{C}$ in a humidified incubator with $95 \%$ air and $5 \% \mathrm{CO}_{2}$ to reach between 70 and $90 \%$ confluence. The following day, the plasmid DNA $(4 \mu \mathrm{g})$ or siRNA $(100 \mathrm{nM})$ were diluted in Opti-MEM® I Reduced Serum medium (Life Technologies). The diluted DNA or siRNA was then mixed with Oligofectamine ${ }^{\text {TM }}$ for the formation of DNA/siRNA-lipid complexes in a total volume of $250 \mu \mathrm{l}$ Opti-MEM® I Reduced Serum medium. After 10-15 min incubation at room temperature, the mixture was added in to the cell medium (DMEM/F-12 supplemented with $10 \%$ FBS and $10 \%$ penicillin/streptomycin) and the cells were incubated for $48 \mathrm{~h}$ at $37^{\circ} \mathrm{C}$ in a humidified incubator with $95 \%$ air and $5 \% \mathrm{CO}_{2}$. Subsequently, whole-cell lysates were prepared for further analysis using Novex ${ }^{\circledR}$ NP40 Cell Lysis Buffer (Invitrogen Life Technologies), as previously described (7).

Cell viability assays. A total of $1 \times 10^{4}$ cells were seeded into each well of a 48-well plate and incubated overnight. The medium was replaced with fresh medium, with or without Taxol at various concentrations (MDA-MB-231 cells: 0, 5, 10, 20, 40, 80, 120, 160 and $200 \mathrm{nM}$; BT-474 cells: 0, 0.05, $0.1,0.2,0.4,0.8,1.2,1.6,2.0$ and $2.4 \mu \mathrm{M})$ and incubated for $48 \mathrm{~h}$ at $37^{\circ} \mathrm{C}$ in a humidified incubator with $95 \%$ air and $5 \%$
$\mathrm{CO}_{2}$. The cell viability was measured using a 3-(4,5 dimethylthiazol-2-yl)-2,5-diphenyltetrazolium bromide assay (Life Technologies). The absorbance was measured spectrophotometrically at $570 \mathrm{~nm}$ using a Universal Microplate Reader EL800 (Bio-Tek instruments, Inc., Vermont, MA, USA).

Glutamine uptake assay. A glutamine uptake assay was performed using a Glutamine and Glutamate Determination kit (GLN1-1KT; Sigma-Aldrich), according to the manufacturer's instructions. Briefly, MDA-MB-231 and BT474 cells with or without taxol treatments; MDA-MB-231 parental and taxol resistant cells; BT474 siGLS1 and control siRNA cells $\left(2 \times 10^{5} /\right.$ well $)$ were plated into 6 -well plates for $24 \mathrm{~h}$ at $37^{\circ} \mathrm{C}$ in a humidified incubator with $95 \%$ air and $5 \% \mathrm{CO}_{2}$. The cell lysates were collected and $10 \mu \mathrm{g}$ total protein of each sample was diluted to $250 \mu 1$, the cell-free supernatant samples were collected and analyzed in triplicate using the glutamine uptake assay kits. The absorbance was measured at $340 \mathrm{~nm}$ for glutamine in a 96-well plate-reader (SpectraMax M2 spectrophotometer; Molecular Devices, Sunnyvale, CA, USA). All values were normalized to the total protein from each well.

Reverse transcription quantitative polymerase chain reaction (RT-qPCR). The total RNA from MDA-MB-231 and BT474 cells under taxol treatments and the taxol-resistant and parental MDA-MB-231 cells was extracted following homogenization of the cells and tissues (QIAshredder; cat. no. 79656; Qiagen, Germantown, MD, USA) using an RNeasy Mini kit (Qiagen) and performing DNase digestion (RNase free DNase kit; Qiagen) during the RNA extraction. The total RNA $(1 \mu \mathrm{g})$ was reverse transcribed using a High Capacity cDNA Reverse Transcription kit (Applied Biosystems, Foster City, CA, USA). The cDNA reaction was diluted to $1: 10$ with $\mathrm{ddH}_{2} \mathrm{O}$ for use as the template for qPCR. TaqMan Gene Expression Assays primers (Life Technologies) and GLS1 specific probes were used for expression analysis and primers and probes against $18 \mathrm{~S}$ ribosomal RNA (Applied Biosystems) were used as internal controls. The qPCR amplifications were performed in a final reaction volume of $10 \mu \mathrm{l}$ containing, 5.5 $\mu \mathrm{l}$ TaqMan Universal PCR Master mix (Applied Biosystems), $0.5 \mu \mathrm{l}$ primers and probes mix and $4.5 \mu \mathrm{g}$ cDNA diluted solution. Primer sequences used were as follows: 18S rRNA forward, 5'-TGCTGTCCCTGTATGCCTCT-3' and reverse, 5'-TGTAGCCACGCTCGGTCA-3'. The cycling conditions were as follows: $2 \mathrm{~min}$ at $50^{\circ} \mathrm{C}, 10 \mathrm{~min}$ at $95^{\circ} \mathrm{C}, 40$ cycles of denaturation for $15 \mathrm{sec}$ at $95^{\circ} \mathrm{C}$ and annealing/extension for $1 \mathrm{~min}$ at $60^{\circ} \mathrm{C}$. The reactions were performed using the Step 1 Plus Real-Time PCR system thermocycler (Applied Biosystems) and all qPCR reactions were performed in triplicate and repeated at least twice. The comparative threshold cycle $(\mathrm{Ct})$ for the mRNA expression was calculated relative to the $\mathrm{Ct}$ of $18 \mathrm{~S}$ ribosomal RNA. The relative mRNA expression was calculated using the $2^{-\Delta \Delta C t}$ method (7). The GLS1 primers used for qPCR were as follows: Forward 5'-CTTTCCATGTTGGTCTTCC-3' and reverse 5'-AAACAAGATCGTGACAAAAGTGAA-3'.

Western blot analysis. The cells $\left(5 \times 10^{5 / 300 ~} \mu 1\right.$ lysis buffer) were lysed in $1 X$ SDS sample buffer. The proteins (40 $\mu \mathrm{g} /$ well) were subsequently resolved by electrophoresis using SDS-PAGE (Novex ${ }^{\circledR} 4-20 \%$ Tris-Glycine Mini 
A

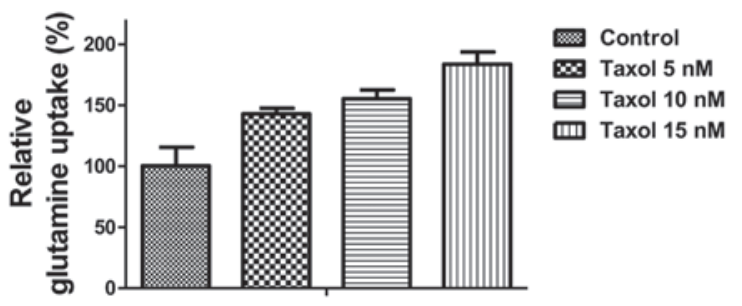

B

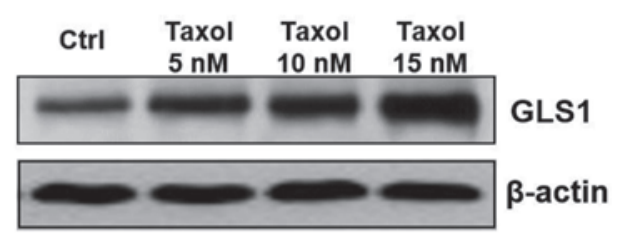

C MDA-MB-231 GLS1 mRNA

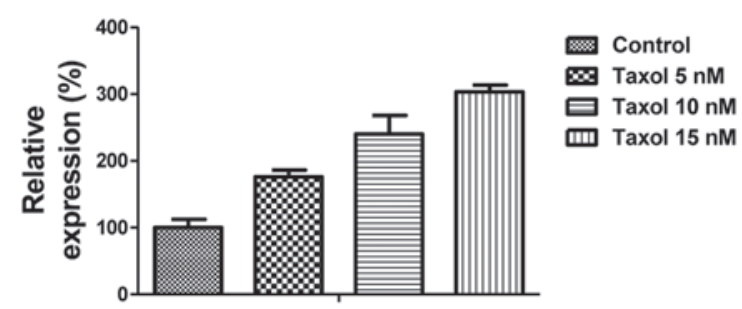

BT -474

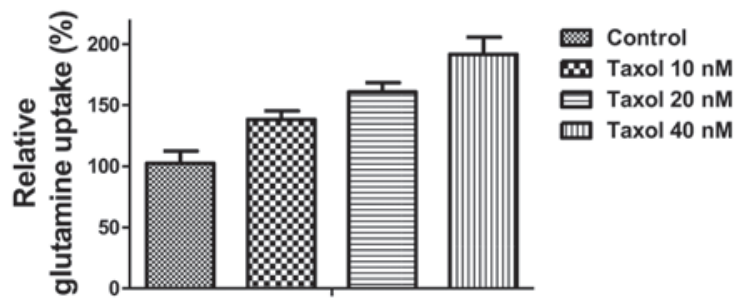

BT-474

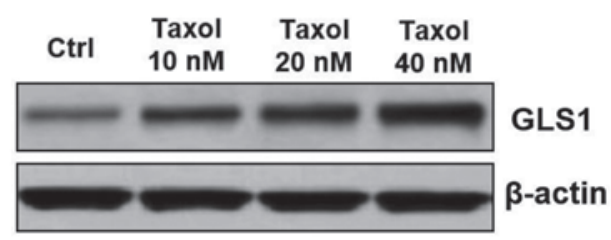

BT-474

GLS1 mRNA

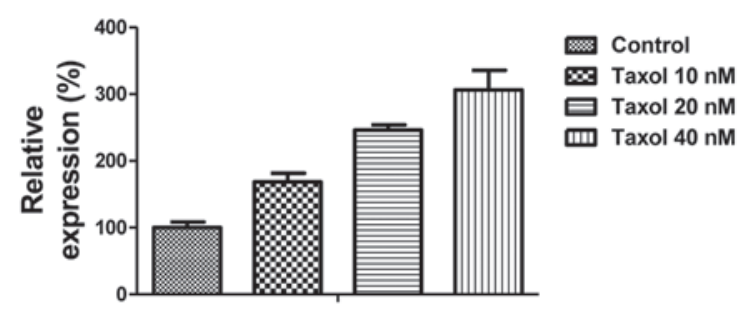

Figure 1. Glutamine uptake and the expression of GLS1 are upregulated in breast cancer cells following treatment with Taxol. (A) Glutamine uptake was induced following treatment with Taxol at different concentrations for $24 \mathrm{~h}$ in MDA-MB-231 and BT-474 breast cancer cells. (B) Western blotting results revealed the protein expression levels of GLS1 in MDA-MB-231 and BT-474 cells following treatment with Taxol at different concentrations. $\beta$-actin was used as a loading control. (C) Reverse transcription quantitative polymerase chain reaction results revealed the mRNA expression levels of GLS1 in MDA-MB-231 and BT-474 cells following treatment with Taxol at different concentrations. The data are expressed as the mean \pm standard error of the mean of three independent experiments. Ctrl, control; GLS, glutaminase.

Gels; Life Technologies) and transferred onto nitrocellulose membranes (Life Technologies). The membranes were probed with primary antibodies overnight at $37^{\circ} \mathrm{C}$, followed by incubating with the appropriate horseradish peroxidase-conjugated secondary antibodies for $2 \mathrm{~h}$ at room temperature prior to detection using a Super Signal Enhanced Chemiluminescence kit (Pierce Biotechnology, Inc., Rockford, IL, USA). For sequential blotting, the membranes were stripped using Restore Western Blot Stripping Buffer (Pierce Biotechnology, Inc.) and re-probed with the appropriate antibodies.

Statistical analysis. Statistical analysis was performed using unpaired Student's t-test with GraphPad Prism 5.0 software (GraphPad Software, Inc., La Jolla, CA, USA). The data are expressed as the mean and $\mathrm{P}<0.05$ was considered to indicate a statistically significant difference.

\section{Results}

Glutamine metabolism and the expression of GLS1 are induced by treatment with Taxol. A previous study demonstrated that Taxol-resistant breast cancer cells exhibited elevated levels of glucose metabolism (18), suggesting that there is an association between cellular metabolism and Taxol-induced cell apoptosis. The present study examined whether treatment with Taxol regulated glutamine metabolism in breast cancer cells. Notably, a significant upregulation of glutamine metabolism was observed following treatment with Taxol at multiple concentrations (Fig. 1A). The MDA-MB-231 and BT-474 (Fig. 1A) cells exhibited an increased glutamine uptake following treatment with Taxol at low-toxic concentrations (MDA-MB-231: 5, 10 and $15 \mathrm{nM}$; BT474: 10, 20 and 40 $\mathrm{nM}$ ) for $48 \mathrm{~h}$. Since the first stage of glutamine metabolism requires GLS1 to generate glutamate (15), the expression of GLS1 following treatment with Taxol was assessed. The results revealed that the protein and mRNA expression of GLS1 were significantly upregulated by low-toxic treatment with Taxol (Fig. 1B and C). This suggested that dysregulated glutamine metabolism and GLS1 may be therapeutic targets for Taxol-induced cancer cell apoptosis.

Taxol-resistant breast cancer cells exhibit upregulated glutamine metabolism and expression of GLS1. To investigate the mechanisms underlying Taxol-induced glutamine metabolism in breast cancer cells, Taxol-resistant cells originating from MDA-MB-231 were isolated by gradually 
A

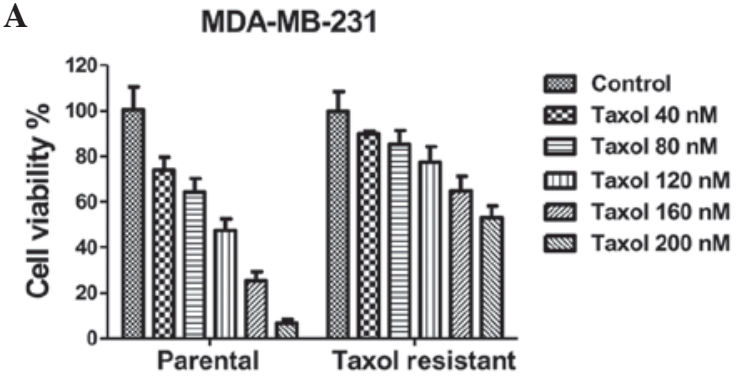

C

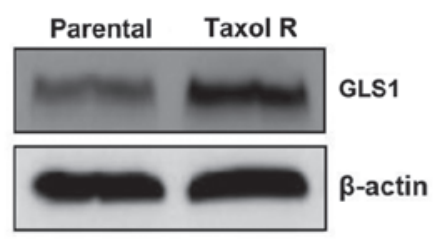

B

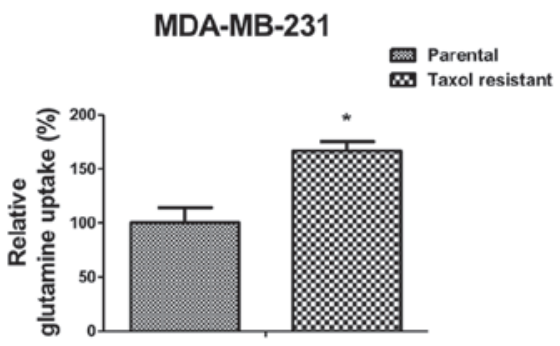

D

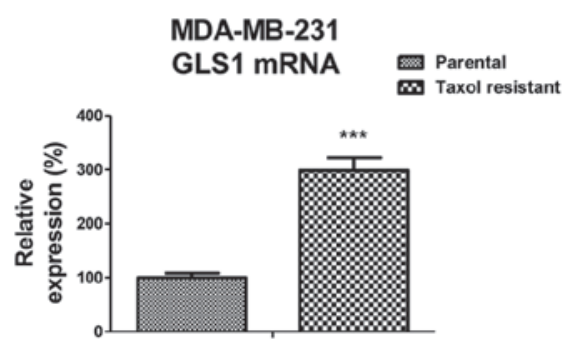

Figure 2. Taxol-resistant breast cancer cells exhibit increased levels of glutamine metabolism and expression of GLS1. (A) Taxol-resistant cells were generated from MDA-MB-231 cells by treating parental cells with gradually increasing concentrations of Taxol in regular cell culture conditions for selection of Taxol-resistant cells. The cell viability of Taxol-resistant cells was analyzed using a 3-(4,5 dimethylthiazol-2-yl)-2,5-diphenyltetrazolium bromide assay following treatment with Taxol at different concentrations for $48 \mathrm{~h}$. (B) Glutamine uptake was measured in the MDA-MB-231 parental cells and the MDA-MB-231 Taxol-resistant cells. (C) Western blotting demonstrated that the expression of GLS1 was upregulated in the MDA-MB-231 Taxol-resistant cells compared with the parental cells. $\beta$-actin was used as a loading control. (D) Reverse transcription quantitative polymerase chain reaction revealed that the mRNA expression of GLS1 was upregulated in the MDA-MB-231 Taxol-resistant cells compared with the parental cells. The data are expressed as the mean \pm standard error of the mean of three independent experiments ( $\mathrm{P}<0.05$ and ${ }^{* * *} \mathrm{P}<0.001$ vs. parental cells). GLS, glutaminase; Taxol R, Taxol-resistant.

A

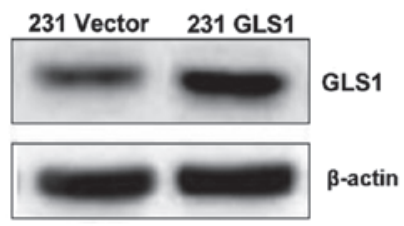

B

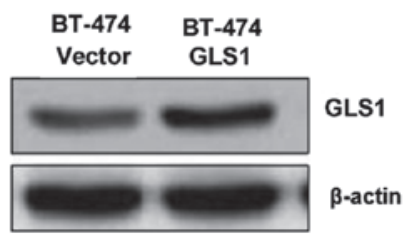

MDA-MB-231
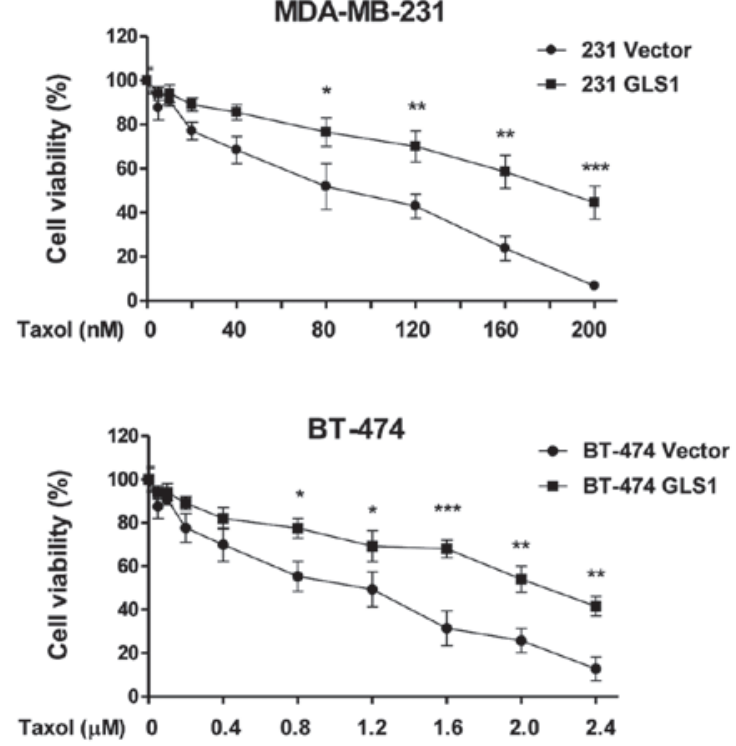

Figure 3. Overexpression of GLS1 contributes to Taxol resistance in breast cancer cells. (A) Transient transfection of an overexpression vector containing wild-type GLS1 into (A) MDA-MB-231 and (B) BT- 474 cells, followed by western blotting. $\beta$-actin was used as a loading control. Following $48 \mathrm{~h}$ transfection, the MDA-MB-231 and BT-474 cells expressing either the vector control or overexpressing GLS1, were treated with Taxol at different concentrations and cell viability was assessed using a 3-(4,5 dimethylthiazol-2-yl)-2,5-diphenyltetrazolium bromide assay. The data are expressed as the mean \pm standard error of the mean of three independent experiments ( $\mathrm{P}<0.05,{ }^{* *} \mathrm{P}<0.01$ and ${ }^{* * * *} \mathrm{P}<0.001$ vs. control transfection). 231, MDA-MB-231 cells; GLS, glutaminase.

treating parental cells with increasing concentrations of Taxol for 8 weeks. The Taxol-resistant MDA-MB-231 colonies were pooled as the Taxol-resistant cells. As shown in Fig. 2A, the Taxol-resistant MDA-MB-231 cells were insensitive to regular Taxol treatments compared with the parental cells. The half maximal inhibitory concentration $\left(\mathrm{IC}_{50}\right)$ of the parental cells was $\sim 100 \mathrm{nM}$, while the $\mathrm{IC}_{50}$ of the Taxol-resistant cells was increased to $\sim 300 \mathrm{nM}$. The glutamine metabolism in Taxol-sensitive and resistant cells were compared. The glutamine uptake was significantly upregulated in the Taxol-resistant cells compared with the Taxol-sensitive cells (Fig. 2B). Consistently, the protein and mRNA expression levels of GLS1 were upregulated in the Taxol-resistant cells (Fig. 2C and D). These findings 
A

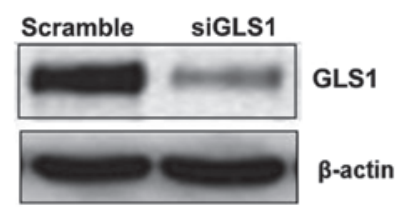

B

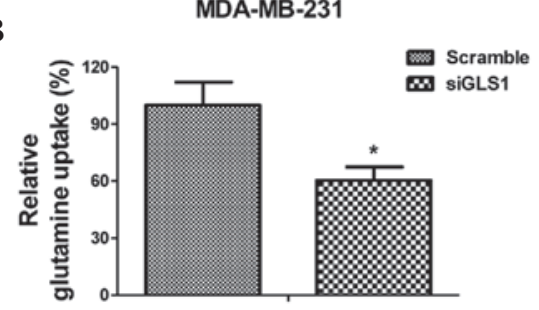

BT -474
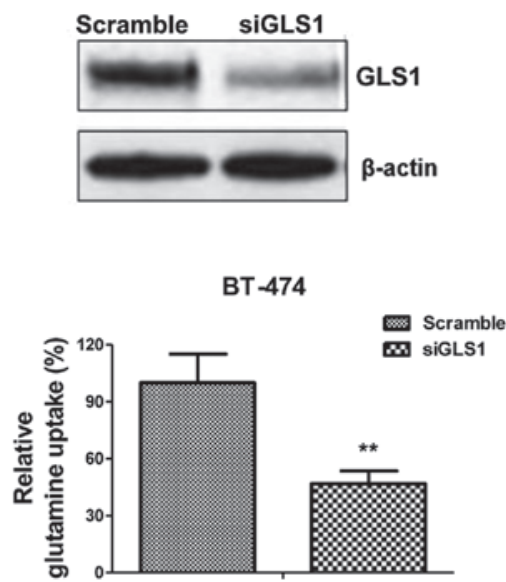

Figure 4. Knock down of GLS1 using siRNA inhibits glutamine metabolism. (A) Knock down of GLS1 using siRNA in MDA-MB-231 and BT-474 cells followed by western blotting revealed the protein expression levels of GLS1, compared with the siRNA-transfected control. (B) Knock down of GLS1 using siRNA in MDA-MB-231 and BT-474 cells followed by measurements of glutamine uptake, compared with the siRNA-transfected control. Data are expressed as the mean \pm standard error of the mean of three independent experiments ( $\mathrm{P}<0.05$ and ${ }^{* *} \mathrm{P}<0.01$ vs. siRNA-transfected control). si, small interfering; GLS, glutaminase; Scramble, control siRNA.

A

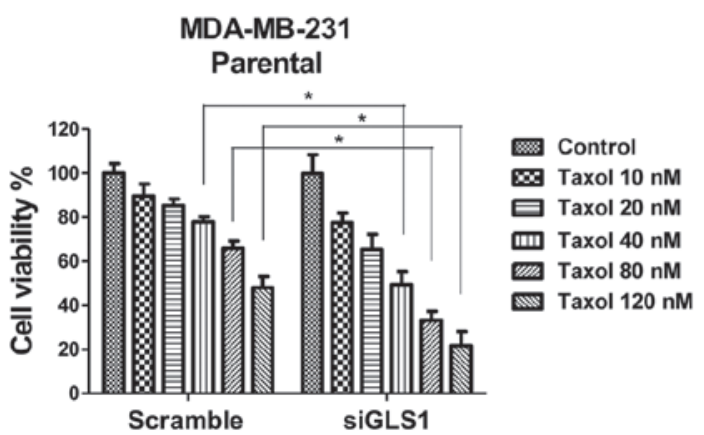

B

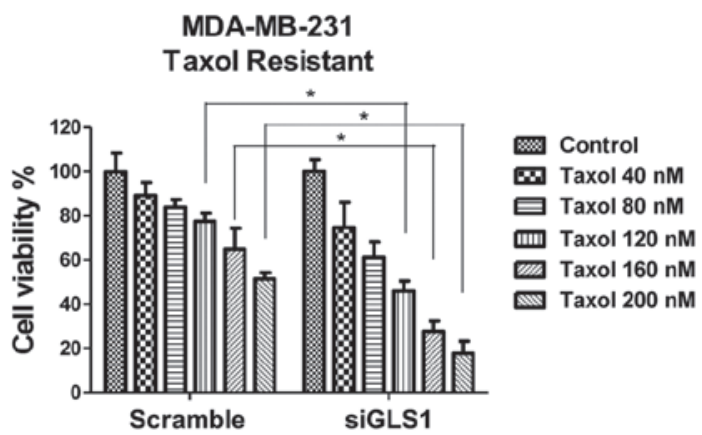

Figure 5. Knock down of GLS1 resensitizes Taxol-resistant breast cancer cells to Taxol. (A) MDA-MB-231 parental cells were transfected with siGLS1 or control siRNA for $48 \mathrm{~h}$, followed by treatment with Taxol at different concentrations. Cell viability was subsequently analyzed using a 3-(4,5 dimethylthiazol-2-yl)-2,5-diphenyltetrazolium bromide assay. (B) Taxol-resistant MDA-MB-231 cells were transfected with siGLS1 or control siRNA for $48 \mathrm{~h}$ followed by treatment with Taxol at different concentrations. Cell viability was analyzed . The data are expressed as the mean \pm standard error of the mean of three independent experiments ("P<0.05 vs. control siRNA). si, small interfering; GLS, glutaminase.

suggested that upregulated glutamine metabolism may be a possible mechanism underlying Taxol resistance in breast cancer cells.

Exogenous overexpression of GLS1 renders breast cancer cells resistant to Taxol. The results suggested that GLS1 may be involved in the Taxol resistance exhibited by breast cancer cells. To further examine the function of GLS1 in Taxol resistance, an overexpression vector, containing WT GLS1, was transiently transfected into the MDA-MB-231 and BT-474 cells (Fig. 3A and $B$ ). The sensitivities of the cells overexpressing GLS1 following treatment with Taxol were compared with the empty vector-transfected cells. Notably, the breast cancer cells overexpressing GLS1 exhibited increased resistance to treatment with Taxol compared with the control cells (Fig. 3A and B). The MDA-MB-231 cells overexpressing GLS1 demonstrated an $\mathrm{IC}_{50}$ of $200 \mathrm{nM}$, compared with control cells, with an $\mathrm{IC}_{50}$ of $100 \mathrm{nM}$. Similarly, the $\mathrm{IC}_{50}$ of the BT-474 control cells was $1.2 \mu \mathrm{M}$, while the $\mathrm{IC}_{50}$ of the Taxol-resistant BT-474 cells was $2.2 \mu \mathrm{M}$. These results further supported that upregulated expression of GSL1 contributed to Taxol resistance in breast cancer cells.
Inhibition of GLS1 resensitizes Taxol-resistant cancer cells to Taxol. The results revealed that treatment with Taxol induced glutamine metabolism and that Taxol-resistant breast cancer cells exhibited upregulated glutamine metabolism and expression of GLS1. The present study, therefore, hypothesized that the combination of GLS1 inhibition and treatment with Taxol may exhibit a synergistic effect to overcome Taxol resistance through the inhibition of glutamine metabolism. Knock down of GLS1 by siRNA significantly inhibited the metabolism of glutamine in the MDA-MB-231 and BT-474 cells (Fig. 4A and B). The glutamine uptake of the MDA-MD-231 and BT-474 cells decreased by 40 and $50 \%$, respectively, compared with the siRNA-transfected control (Fig. 4B). The present study subsequently examined whether GLS1 knockdown using siRNA in Taxol-resistant cells resulted in re-sensitization of the cells to Taxol. The expression of GLS1 was reduced using siRNA in the MDA-MB-231 parental cells and the Taxol-resistant cells, followed by treatment with Taxol at different concentrations for $48 \mathrm{~h}$. The data revealed that knock-down of GLS1 in the parental cells and the Taxol-resistant cells rendered them sensitive to Taxol (Fig. 5). Cells with a lower expression of 
GLS1 exhibited reduced viability following treatment with Taxol. These results supported the hypothesis that inhibition of glutamine metabolism by knocking down GLS1 resensitizes Taxol-resistant cancer cells to Taxol.

\section{Discussion}

The 'Warburg effect' describes the characteristic of cancer cells to produce energy predominantly from the glycolytic breakdown of glucose, rather than mitochondrial oxidative phosphorylation (13). In addition, cancer cells exhibit other metabolic characteristics, including increased fatty acid synthesis and glutamine metabolism (13). Glutamine, the most abundant amino acid in the blood, is important in cell growth and metabolism. Cancer cells rely on glutamine metabolism for increased production of by-products, which are necessary for rapidly proliferating cells, including amino-acid precursors (16). Additionally, glutaminolysis represents a fundamental mechanism for nitrogen anabolism $(16,19)$.

It has been reported that glutaminolysis is associated with drug resistance via the activation of mammalian target of rapamycin complex 1 signaling in gastric cancer (20). The present study demonstrated that glutamine metabolism was induced by treatment with Taxol in human breast cancer cells. According to this phenotype, Taxol-resistant cells were established from the MDA-MB-231 cell line, and the results revealed that the Taxol-resistant cells exhibited upregulated glutamine uptake rate compared with the parental cells. This indicated that dysregulated glutamine metabolism may be a target for the development of therapeutic drugs for overcoming Taxol resistance in patients with cancer.

The first stage of glutamine metabolism involves GLS catalyzing the conversion of glutamine to glutamate. The present study revealed that the expression of GLS1 was upregulated in Taxol-resistant cells. It has been reported that the overexpression of GLS1 correlates with cell proliferation and tumor growth (21). Inhibition of GLS prevents oncogenic transformation and slows cell growth in certain types of glioma (22). The present investigation demonstrated that the overexpression of GLS1 rendered breast cancer cells resistant to Taxol, suggesting a close association between GLS1 and Taxol resistance. Inhibition of the expression of GLS1 using siRNA significantly resensitized Taxol-resistant cells to Taxol, indicating that, overexpression of GLS1 may be the mechanism underlying Taxol resistance in cancer cells. It is possible that tumor-initiating oncogenes may promote glutamine utilization through elevating the expression of GLS as part of the metabolic transformation process. Overexpressed oncogenes, including c-Myc, have been demonstrated to increase the expression of GLS1 in human tumor cells (23). In addition, ERBB2 has been suggested to contribute to Taxol resistance by promoting glycolysis in cancer cells (10). A previous investigation reported that the activation of ERBB2 upregulates the expression of GLS1, which promotes the proliferation of breast cancer cells (24). This indicates that upregulated expression levels of GLS1 and glutamine metabolism may be the mechanism underlying ERBB2-induced Taxol resistance. Further investigation aims to examine the detailed mechanism of Taxol-induced glutamine metabolism in cancer cells and identify inhibitors of glutamine metabolism for the development of therapeutic strategies to overcoming drug resistance.

\section{Acknowledgements}

The authors would like to thank the staff and faculty of the Department of Medical Oncology, Yantai Yantaishan Hospital (Yhina, China), including Dr Yaobo Song for their editorial assistance.

\section{References}

1. Henley D, Isbill M, Fernando R, Foster JS and Wimalasena J: Paclitaxel induced apoptosis in breast cancer cells requires cell cycle transit but not Cdc2 activity. Cancer Chemother Pharmacol 59: 235-249, 2007.

2. Tan M and Yu D: Molecular mechanisms of ERBB2-mediated breast cancer chemoresistance. Adv Exp Med Biol 608: 119-129, 2007.

3. Orr GA, Verdier-Pinard P, McDaid H and Horwitz SB: Mechanisms of Taxol resistance related to microtubules. Oncogene 22: 7280-7295, 2003.

4. Frankel A, Buckman R and Kerbel RS: Abrogation of taxol-induced G2-M arrest and apoptosis in human ovarian cancer cells grown as multicellular tumor spheroids. Cancer Res 57: 2388-2293, 1997.

5. Yin S, Bhattacharya R and Cabral F: Human mutations that confer paclitaxel resistance. Mol Cancer Ther 9: 327-335, 2010.

6. Zaffaroni N, Pennati M, Colella G, Perego P, Supino R, Gatti L, Pilotti S, Zunino F and Daidone MG: Expression of the anti-apoptotic gene survivin correlates with taxol resistance in human ovarian cancer. Cell Mol Life Sci 59: 1406-1412, 2002.

7. Wertz IE, Kusam S, Lam C, Okamoto T, Sandoval W, Anderson DJ, Helgason E, Ernst JA, Eby M, Liu J, Belmont LD, Kaminker JS, O'Rourke KM, Pujara K, Kohli PB, Johnson AR, Chiu ML, Lill JR, Jackson PK, Fairbrother WJ, Seshagiri S, Ludlam MJ, Leong KG, Dueber EC, Maecker H, Huang DC and Dixit VM: Sensitivity to antitubulin chemotherapeutics is regulated by MCL1 and FBW7. Nature 471: 110-114, 2011.

8. Ferlini C, Cicchillitti L, Raspaglio G, Bartollino S, Cimitan S, Bertucci C, Mozzetti S, Gallo D, Persico M, Fattorusso C, Campiani G and Scambia G: Paclitaxel directly binds to Bcl-2 and functionally mimics activity of Nur77. Cancer Res 69: 6906-6914, 2009

9. Yu D, Liu B, Jing T, Sun D, Price JE, Singletary SE, Ibrahim N, Hortobagyi GN and Hung MC: Overexpression of both p185c-ERBB2 and p170mdr-1 renders breast cancer cells highly resistant to taxol. Oncogene 16: 2087-2094, 1998.

10. Ding Y, Liu Z, Desai S, Zhao Y, Liu H, Pannell LK, Yi H, Wright ER, Owen LB, Dean-Colomb W, Fodstad O, Lu J, LeDoux SP, Wilson GL and Tan M: Receptor tyrosine kinase ERBB2 translocates into mitochondria and regulates cellular metabolism. Nat Commun 3: 1271, 2012.

11. Kim SH, Juhnn YS and Song YS: Akt involvement in paclitaxel chemoresistance of human ovarian cancer cells. Ann NY Acad Sci 1095: 82-89, 2007.

12. Chen T, Pengetnze Y and Taylor CC: Src inhibition enhances paclitaxel cytotoxicity in ovarian cancer cells by caspase-9-independent activation of caspase-3. Mol Cancer Ther 4: 217-224, 2005.

13. Zhao Y, Butler EB and Tan M: Targeting cellular metabolism to improve cancer therapeutics. Cell Death Dis 4: e532, 2013.

14. Aledo JC, Gómez-Fabre PM, Olalla L and Marquez J: Identification of two human glutaminase loci and tissue-specific expression of the two related genes. Mamm Genome 11: 1107-1110, 2000.

15. Gómez-Fabre PM, Aledo JC, Del Castillo-Olivares A, Alonso FJ, Nunez De Castro I, Campos JA and Marquez J: Molecular cloning, sequencing and expression studies of the human breast cancer cell glutaminase. Biochem J 345: 365-375, 2000.

16. Dang CV: Glutaminolysis: supplying carbon or nitrogen or both for cancer cells? Cell Cycle 9: 3884-3886, 2010.

17. Wise DR and Thompson CB: Glutamine addiction: a new therapeutic target in cancer. Trends Biochem Sci 35: 427-433, 2010 .

18. Zhou M, Zhao Y, Ding Y, Liu H, Liu Z, Fodstad O, Riker AI, Kamarajugadda S, Lu J, Owen LB, Ledoux SP and Tan M: Warburg effect in chemosensitivity: targeting lactate dehydrogenase-A re-sensitizes taxol-resistant cancer cells to taxol. Mol Cancer 9: 33, 2010. 
19. Meng M, Chen S, Lao T, Liang D and Sang N: Nitrogen anabolism underlies the importance of glutaminolysis in proliferating cells. Cell Cycle 9: 3921-3932, 2010.

20. Durán RV, Oppliger W, Robitaille AM, Heiserich L, Skendaj R, Gottlieb E, et al: Glutaminolysis activates Rag-mTORC1 signaling. Mol Cell 47: 349-358, 2012.

21. de la Rosa V, Campos-Sandoval JA, Martin-Rufian M, Cardona C, Mates JM, Segura JA, Alonso FJ and Marquez J: A novel glutaminase isoform in mammalian tissues. Neurochem Int 55: 76-84, 2009.

22. Seltzer MJ,BennettBD, Joshi AD, Gao P, Thomas AG, Ferraris DV, Tsukamoto T, Rojas CJ, Slusher BS, Rabinowitz JD, et al: Inhibition of glutaminase preferentially slows growth of glioma cells with mutant IDH1. Cancer Res 70: 8981-8987, 2010.
23. Gao P, Tchernyshyov I, Chang TC, Lee YS, Kita K, Ochi T, Zeller KI, De Marzo AM, Van Eyk JE, Mendell JT and Dang CV: c-Myc suppression of miR-23a/b enhances mitochondrial glutaminase expression and glutamine metabolism. Nature 458: 762-765, 2009.

24. Qie S, Chu C, Li W, Wang C and Sang N: ERBB2 activation upregulates glutaminase 1 expression which promotes breast cancer cell proliferation. J Cell Biochem 115: 498-509, 2014. 